\title{
Identification of Epithelial Mesenchymal Transition-Related lncRNAs Associated with Prognosis and Tumor Immune Microenvironment of Hepatocellular Carcinoma
}

\author{
Yongjie Zhou $\mathbb{D}^{1,2,3}$ Liangwen Wang, ${ }^{1,2,3}$ Wen Zhang, ${ }^{1,2,3}$ Jingqin Ma, ${ }^{1,2,3}$ Zihan Zhang, ${ }^{1,2,3}$ \\ Minjie Yang, ${ }^{1,2,3}$ Jiaze Yu, ${ }^{1,2,3}$ Jianjun Luo $\mathbb{D}^{1,2,3,4}$ and Zhiping Yan $\mathbb{D}^{1,2,3,4}$ \\ ${ }^{1}$ Department of Interventional Radiology, Zhongshan Hospital, Fudan University, Shanghai, China \\ ${ }^{2}$ Shanghai Institution of Medical Imaging, Shanghai, China \\ ${ }^{3}$ National Clinical Research Center for Interventional Medicine, Zhongshan Hospital, Fudan University, Shanghai, China \\ ${ }^{4}$ Center for Tumor Diagnosis and Therapy, Jinshan Hospital, Fudan University, Shanghai, China
}

Correspondence should be addressed to Jianjun Luo; luo.jianjun@zs-hospital.sh.cn

and Zhiping Yan; yan.zhiping@zs-hospital.sh.cn

Yongjie Zhou and Liangwen Wang contributed equally to this work.

Received 26 September 2021; Accepted 24 December 2021; Published 15 January 2022

Academic Editor: Yan-Ming Xu

Copyright (C) 2022 Yongiie Zhou et al. This is an open access article distributed under the Creative Commons Attribution License, which permits unrestricted use, distribution, and reproduction in any medium, provided the original work is properly cited.

Purpose. The long noncoding RNAs (lncRNAs) play the important role in tumor occurrence and progression, and the epithelial to mesenchymal transition (EMT) is the critical process for tumor migration. However, the role of EMT-related lncRNA in hepatocellular carcinoma (HCC) has not been elucidated. Methods. In this study, we selected the EMT-related lncRNAs in HCC by using data from The Cancer Genome Atlas database (TCGA). Two prognostic models of the overall survival (OS) and relapse-free survival (RFS) were constructed and validated through Cox regression model, Kaplan-Meier analysis, and the receiver-operating characteristic (ROC) curves. The unsupervised clustering analysis was utilized to investigate the association between EMT-lncRNAs with tumor immune microenvironment. ESTIMATE algorithm and gene set enrichment analysis (GSEA) were used to estimate tumor microenvironment and associated KEGG pathways. Results. Two EMT-related lncRNA prognostic models of OS and RFS were constructed. Kaplan-Meier curves showed the dismal prognosis of OS and RFS in the group with high-risk score. The ROC curves and AUC values in two prognostic models indicated the discriminative value in the training set and validation set. Patients with HCC were clustered into two subgroups according the unsupervised clustering analysis. Lnc-CCNY-1 was selected as the key lncRNA. GSVA analysis showed that lnc-CCNY-1 was negatively associated with peroxisome proliferator-activated receptor (PPAR) signaling pathway and positively correlated with CELL cycle pathway. Conclusion. Two EMT-related lncRNA prognostic models of OS and RFS were constructed to discriminate patients and predict prognosis of HCC. EMT-related lncRNAs may play a role on prognosis of HCC by influencing the immune microenvironment. Lnc-CCNY-1 was selected as the key EMT-related lncRNA for further exploration.

\section{Introduction}

Hepatocellular carcinoma (HCC) is the most common cancer and the third leading cause of cancer-related deaths worldwide [1]. Despite great advance had made in the treatment modality for HCC, the prognosis of HCC remains dismal with 1 - year survival $<50 \%$ due to high recurrence rate [2]. The frequent methods for classification and prognosis prediction of patients with HCC were the BarcelonaClinic Liver Cancer (BCLC) staging system [3]. However, it could not precisely predict prognosis of patients with HCC due to high heterogeneous of HCC. It is necessary to construct a novel method to discriminate patients and predict prognosis. 


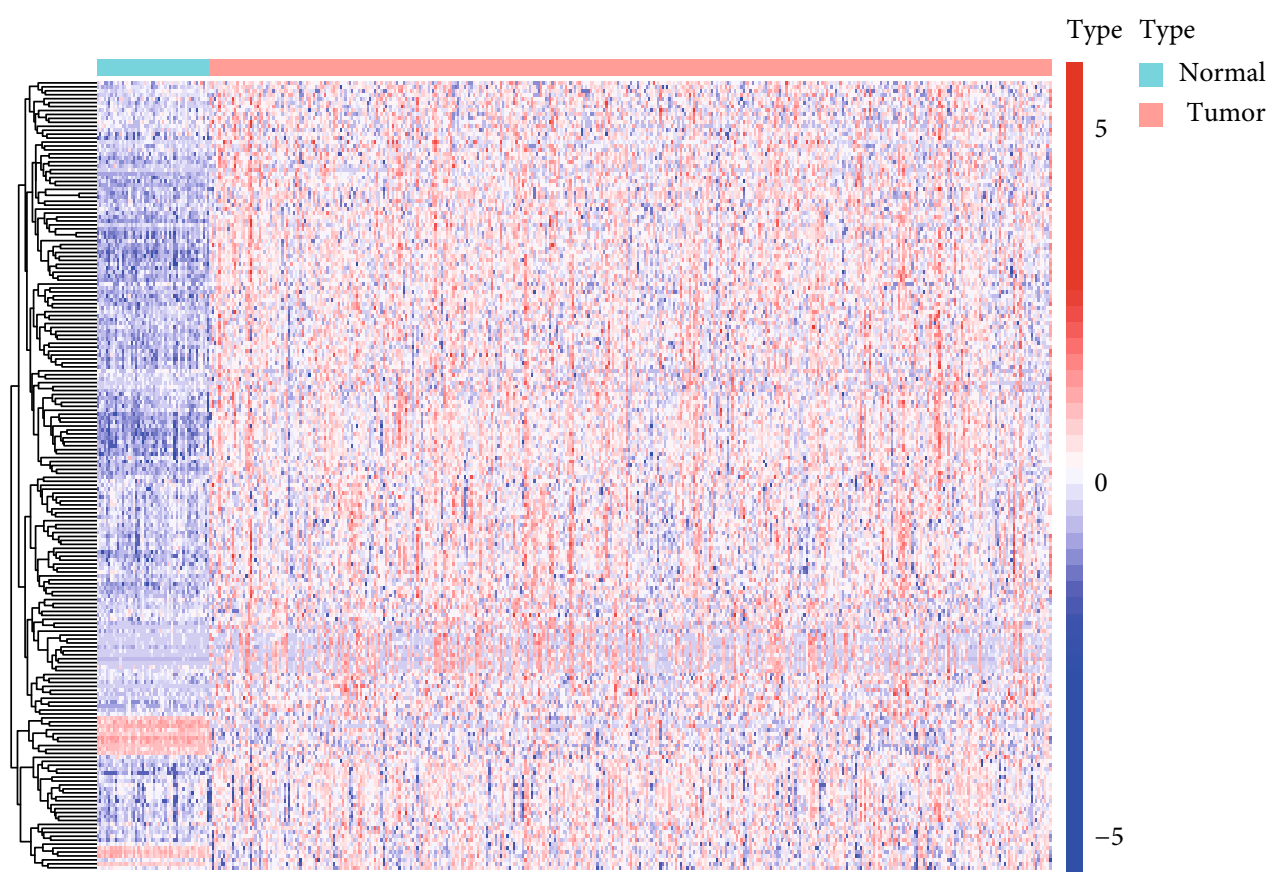

(a)

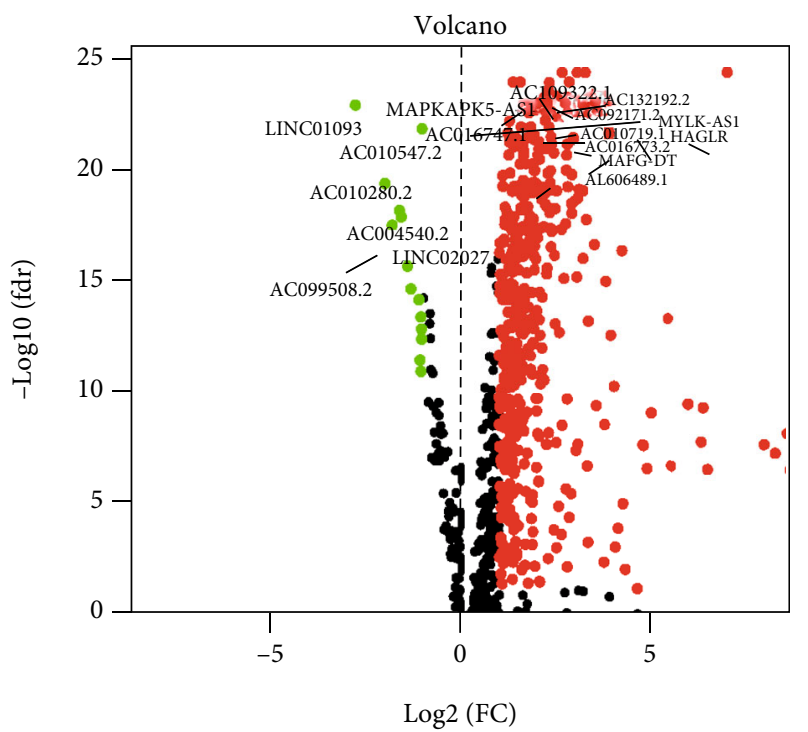

(b)

Figure 1: Identification of differentially expressed EMT-related lncRNAs. The heatmap (a) and the volcano plot (b) showed the differentially expressed EMT-related lncRNAs between HCC tumor tissues and adjacent normal tissues.

The epithelial to mesenchymal transition (EMT) is a multistep biological process, which is characterized by epithelial cell lose polarity and connection to the basement membrane and gains the ability of invasion, migration, and resistance to apoptosis. EMT has been reported to play a crucial role in early steps of metastasis in carcinoma progression [4]. Several studies had reported that various genes regulate EMT to affect tumor process of invasion, metastasis and proliferation in HCC [5-8].

Due to the development of next-generation sequencing technology, the tumor gene characteristics received more attention. LncRNA characterized by greater than $200 \mathrm{nt}$ in length was a form of noncoding RNAs. It was reported that lncRNAs are incorporated in several pathophysiological processes of tumor progression [9-13]. Several studies had demonstrated that lncRNAs were associated with prognosis and HCC proliferation, invasion, and metastasis [14-16]. Therefore, we speculated that EMT-related lncRNA may serve a promising target for predicting prognosis of patients with HCC.

Immunotherapy has made great advances and has been emerged as an effective treatment in HCC and other malignant tumors [17-20]. However, only a small portion of patients with HCC could obtain favorable prognosis from 
immunotherapy. It has been demonstrated that tumor microenvironment (TME) acted a critical role in tumor progression and affected the effect of immunotherapy. TME defined as the environment around a tumor included the surrounding blood vessels, immune cells, adipocytes, mesenchymal stem cells, and the extracellular matrix (ECM) [21, 22]. Several studies had reported that CD8+, CD4+cells, regulatory $\mathrm{T}$ cells, and dendritic cells (DCs) which were located in TME were associated with the efficacy of immunotherapy by influence immune checkpoint inhibitors (ICIs) [23, 24]. However, the identification of Tertiary Lymphoid Structures (TLS) cells is not sufficient to characterize the complex tumor immune milieu. Then, it is worth to investigate landscape of immune cells infiltrating the TME of HCC.

In this study, we identified differentially expressed EMTrelated lncRNAs by comparing the tumor samples and the normal samples from The Cancer Genome Atlas database (TCGA) database. Then, the prognostic EMT-related lncRNA models were constructed and validated. The key EMT-related lncRNA which was simultaneously related to overall survival (OS) and disease-free survival (RFS) was selected for further investigation. ESTIMATE and CIBERSORT algorithms were performed to explore the intratumoral immune landscape.

\section{Materials and Methods}

2.1. Patient Datasets. The gene expression profile of HCC patients was downloaded from TCGA-LIHC database, and the clinical information of these patients was retrieved from cBioportal database. We selected a list of lncRNA by using the gene annotation in the GENCODE project. All data were downloaded from public database, so ethical approval and informed consent were waived.

2.2. Differentially Expressed EMT-Related IncRNA. The EMT-related gene profile from EMT gene database (http:// dbemt.bioinfo-minzhao.org/download.cgi) was downloaded. We performed Pearson correlation analysis to select EMTrelated IncRNAs, when the correlation coefficient $|r|>0.4$ and $p<0.05$. We obtained the differentially expressed EMT-related lncRNAs by comparing the tumor samples and the normal samples using the "limma" package in the $\mathrm{R}$ software, with $|\log \mathrm{FC}|>1$ and a false discovery rate $<$ 0.05 .

2.3. Construction and Validation of Prognostic EMT-Related IncRNA Signature. HCC patients were randomly divided into the training set and the validation set with ratio $7: 3$. HCC patients in the training set were used to construct the prognostic signature, while HCC patients in the validation set were performed to verify the established signature. The univariate Cox proportional regression analysis was performed to screen preliminary EMT-related lncRNAs associated with OS and RFS, which were further analyzed using the multivariate Cox proportional hazard regression model. Finally, the prognostic EMT-LncRNAs were identified to calculate risk score. The risk score of each patient with HCC in both training and validation sets was calculated as below
TABLE 1: The clinicopathological characteristics of patients with HCC.

\begin{tabular}{|c|c|}
\hline Characteristics & No. (\%) \\
\hline \multicolumn{2}{|l|}{ Gender } \\
\hline Male & $255(67.6)$ \\
\hline Female & $122(32.3)$ \\
\hline \multicolumn{2}{|l|}{ Age } \\
\hline$\leq 65$ & $236(62.5)$ \\
\hline$>65$ & $141(37.5)$ \\
\hline \multicolumn{2}{|l|}{ Grade } \\
\hline G1-G2 & $236(62.6)$ \\
\hline G3-G4 & $137(36.3)$ \\
\hline Unknown & $4(1.1)$ \\
\hline \multicolumn{2}{|l|}{ Stage } \\
\hline Stage I-II & $262(69.5)$ \\
\hline Stage III-IV & $92(24.4)$ \\
\hline Unknown & $23(6.1)$ \\
\hline \multicolumn{2}{|l|}{$\mathrm{T}$ stage } \\
\hline $\mathrm{T} 1-\mathrm{T} 2$ & $279(74.0)$ \\
\hline $\mathrm{T} 3-\mathrm{T} 4$ & $92(24.4)$ \\
\hline Unknown & $6(1.6)$ \\
\hline \multicolumn{2}{|l|}{$\mathrm{N}$ stage } \\
\hline No & 255 (67.6) \\
\hline N1 & $118(31.3)$ \\
\hline Unknown & $4(1.1)$ \\
\hline \multicolumn{2}{|l|}{ M stage } \\
\hline M0 & $272(72.1)$ \\
\hline M1 & $101(26.8)$ \\
\hline Unknown & $4(1.1)$ \\
\hline
\end{tabular}

formula: $\quad$ Risk score $=\beta \operatorname{lncRNA} 1 \times \operatorname{lncRNA} 1$ Expression $+\beta$ $\ln c$ RNA2 $\times \ln c$ RNA2 Expression $+\beta \ln c$ RNA3 $\times \ln c$ RNA3 Expression $+\cdots+\beta \operatorname{lncRNAn} \times \operatorname{lncRNAn}$ Expression. Here, " $\beta$ " was the regression coefficient which was generated from the multivariate Cox proportional hazard regression analysis.

Based on the median risk score, HCC patient in both sets were allocated into high- or low-risk groups. The KaplanMeier survival curves were performed to compare prognosis of two groups, and the receiver-operating characteristic (ROC) curves at 1,3 , and 5 years were utilized to evaluate the predictive ability of this model. To further analyze prognostic value in different stages of HCC patients, we performed the ROC curves at 1,3 , and 5 years in four stages.

2.4. mRNA-lncRNA Network. We performed the Pearson correlation analysis to explore the interaction between mRNA and EMT-related lncRNA. It was considered significant association, with the criterion of correlation coefficient $|R|>0.4$ and $p<0.005$. Cytoscape was utilized to visualize the coexpression network.

2.5. Correlation of EMT-Related IncRNAs with Immune Tumor Microenvironment. We utilized the unsupervised consensus approach to generate two clusters of HCC cohort 
TABLE 2: EMT-related lncRNAs associated with overall survival (OS).

\begin{tabular}{lccccc}
\hline ID & Coef & HR & HR.95L & HR.95H & $p$ value \\
\hline Inc-CCNY-1 & 0.457342603 & 1.57987006 & 1.250877801 & 1.995390281 & 0.00012358 \\
AC022424.1 & 0.046529762 & 1.047629258 & 1.009914891 & 1.086752034 & 0.012868687 \\
AC079305.2 & 0.034220542 & 1.034812801 & 1.012748546 & 1.05735776 & 0.001858415 \\
AC068506.1 & 0.210820365 & 1.234690542 & 1.104272923 & 1.380510834 & 0.000214405 \\
AC026369.2 & 0.418336152 & 1.519431348 & 1.230999207 & 1.875445255 & $9.82 \mathrm{E}-05$ \\
ELFN1-AS1 & 0.045657717 & 1.046716077 & 1.004076559 & 1.091166341 & 0.03142199 \\
AC009005.1 & 0.140782576 & 1.151174328 & 1.024057994 & 1.294069615 & 0.018364991 \\
AC068580.1 & 0.238162915 & 1.268915901 & 1.066708751 & 1.509453787 & 0.007164066 \\
AL049840.6 & 0.079975567 & 1.0832606 & 1.011253892 & 1.160394572 & 0.022676322 \\
\hline
\end{tabular}

TABLE 3: EMT-related lncRNAs associated with relapse-free survival (RFS).

\begin{tabular}{lccccc}
\hline ID & Coef & HR & HR.95L & HR.95H & $p$ value \\
\hline ZFPM2-AS1 & 0.090455547 & 1.094672845 & 1.040883457 & 1.151241889 & 0.000433751 \\
lnc-CCNY-1 & 0.289126912 & 1.335261178 & 1.04681349 & 1.703190139 & 0.019890581 \\
AC005332.3 & 0.102852689 & 1.108328128 & 1.055492193 & 1.163808931 & $3.67 \mathrm{E}-05$ \\
\hline
\end{tabular}

through "Consensus Cluster Plus" package, to investigate the association between EMT-lncRNAs with tumor immune microenvironment. ESTIMATE algorithm [25] was used to estimate tumor purity and the extent and level of infiltrating cells (stromal cell, immune cell, and ESTIMATE score), which represented the characteristics of tumor immune microenvironment. Subsequently, the Single Sample Gene Set Enrichment Analysis (ssGSEA) algorithm was used to elucidate the enrichment of 29 immune function-related gene sets, and CIBERSORT algorithm was performed to quantify the fraction of 22 types of immune cells in each sample [26]. We correlated clusters with survival prognosis, clinical features, and immune infiltration.

2.6. A Key EMT-Related IncRNA in HCC. Based on the multivariate Cox proportional hazard regression analysis, differentially expressed EMT-related lncRNAs were identified, including for OS signature and for RFS signature. Among them, EMT-related lncRNA lnc-CCNY-1 was both associated with $O S$ and RFS. The Kaplan-Meier survival curves and log-rank test were performed for lncRNAs for further research. The EMT-related lncRNAs which showed the more relevant to prognosis with prognosis of HCC was identified.

Gene Set Variation Analysis (GSVA) was utilized to characterize pathways or signature summaries in samples of expression datasets. Multivariate Cox proportional hazard regression analysis was used to select pathways associated with prognosis, and Pearson correlation analysis was used to investigate the association of prognosis-related pathways with immune microenvironment.

\section{Results}

3.1. Identification of Differentially Expressed EMT-Related IncRNAs. Overall, 374 tumor samples and 50 adjacent normal samples from TCGA-LIHC database were incorporated in our research. We identified EMT-related lncRNAs by using the TCGA database and EMT gene database. Then, the differentially expressed EMT-related lncRNAs were selected by the comparison of tumor tissues with adjacent normal tissues, which included 478 upregulated lncRNAs and 14 downregulated lncRNAs (Supplementary Table S1). The heatmap and volcano plot were showed characteristics of these differentially expressed EMT-related lncRNAs (Figures 1(a) and 1(b)).

3.2. Construction and Validation of Prognostic Models. Based on the criteria, we obtained 377 HCC patients with clinical information from TCGA database. The clinicopathological characteristics of these patients were presented in Table 1.

These 377 HCC patients with HCC were randomly divided into the training set $(n=264)$ and validation set $(n=113)$ with the ratio of $7: 3$. We performed the univariate Cox regression analysis to select the EMT-related lncRNAs, which were associated with prognosis in the training set. 492 lncRNAs were determined for OS signature, and 480 lncRNAs were selected for RFS signature (Supplementary Tables S2, S3). We further conducted multivariate Cox regression to construct the prognostic model, and nine EMT-related lncRNAs for OS prognosis and three EMT-related lncRNAs for RFS prognosis were identified (Tables 2 and 3 ). The risk scores were calculated as follows: OS signature $=1.57987 \times \operatorname{lnc}-\mathrm{CCNY}-1$ expression $+1.04763 \times \mathrm{AC}$ 022424.1 expression $+1.03481 \times$ AC079305.2 expression + $1.23469 \times$ AC068506.1 expression $+1.51943 \times$ AC026369. 2 expression $+1.04672 \times$ ELFN1 - AS1 expression $+1.15117 \times$ AC009005.1 expression $+1.26892 \times$ AC068580.1 expression $+1.08326 \times$ AL049840.6 expression, RFS signature $=1.09467$ $\times$ ZFPM2 - AS1 expression + $1.33526 \times \operatorname{lnc}-\mathrm{CCNY}-1$ expression $+1.10833 \times$ AC005332.3 expression.

Based on the median cut-off value, patients were divided into the high-risk group and low-risk group both in the 


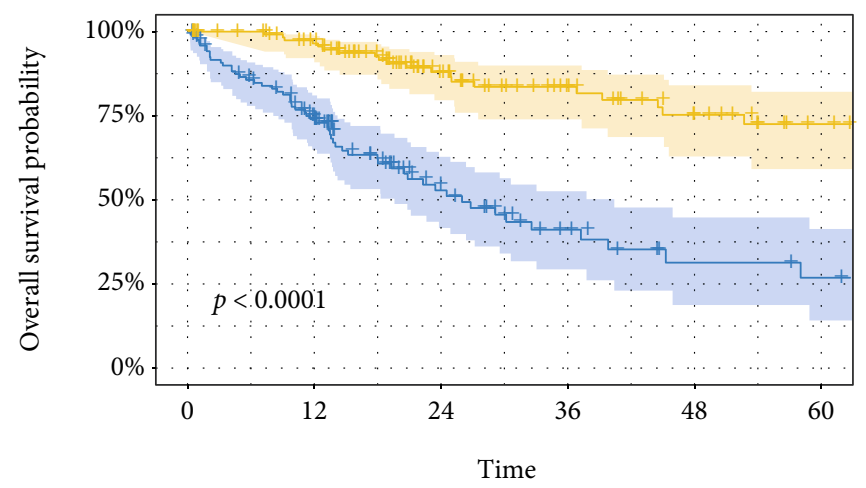

Number at risk

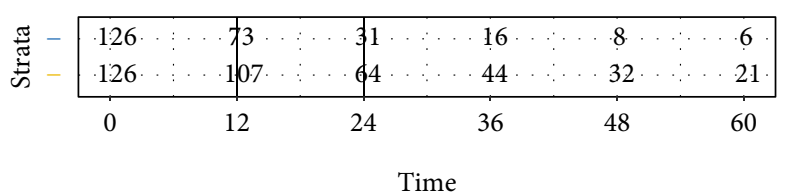

Strata

+ Risk $=$ high

+ Risk = low

(a)

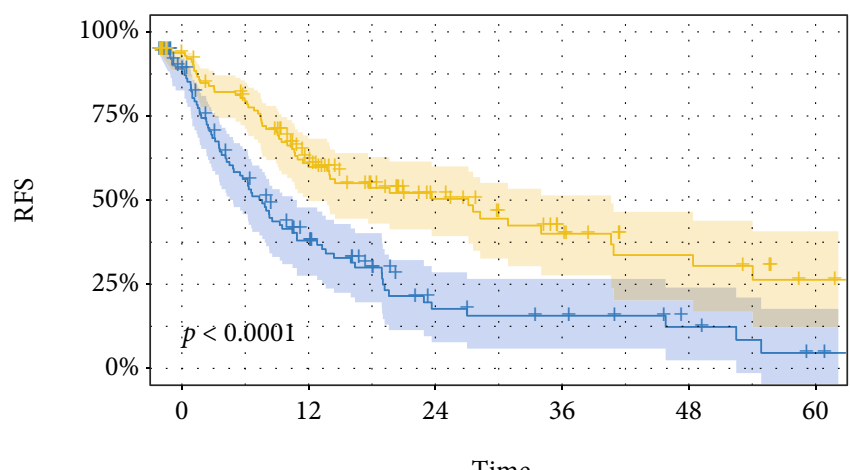

Number at risk

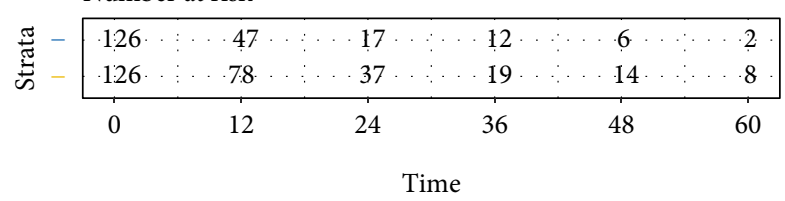

Strata

+ Risk $=$ high

+ Risk = low

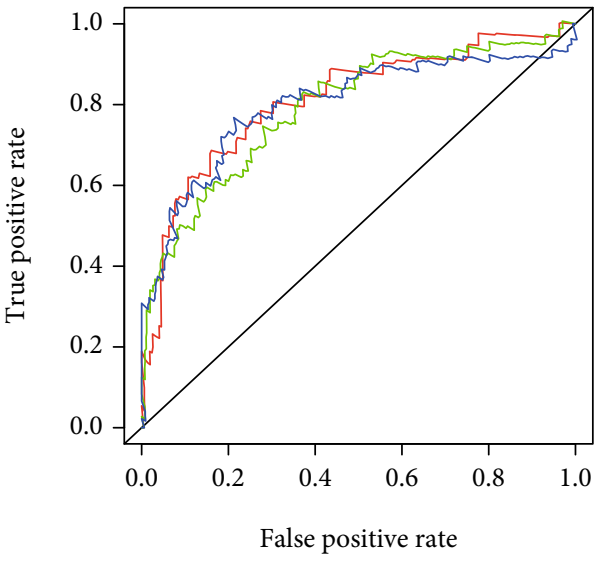

- One year $(\mathrm{AUC}=0.811)$

Two year $(\mathrm{AUC}=0.793)$

Three year $(\mathrm{AUC}=0.800)$

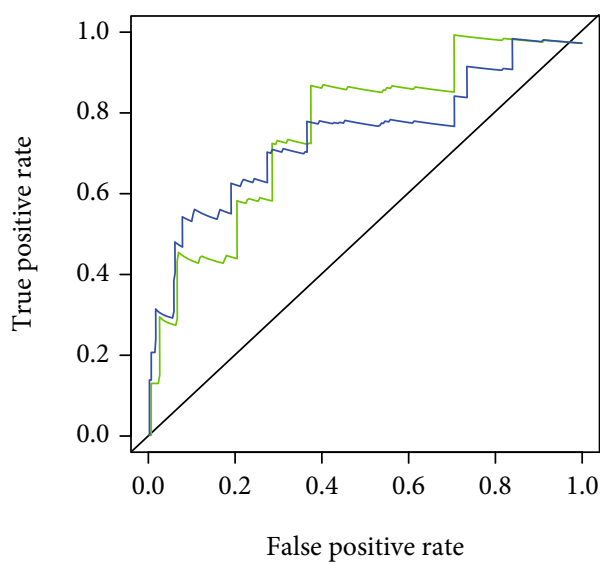

- One year $(\mathrm{AUC}=\mathrm{NaN})$

Two year $(\mathrm{AUC}=0.783$ )

Three year $(\mathrm{AUC}=0.765)$

(b)

Figure 2: Continued. 

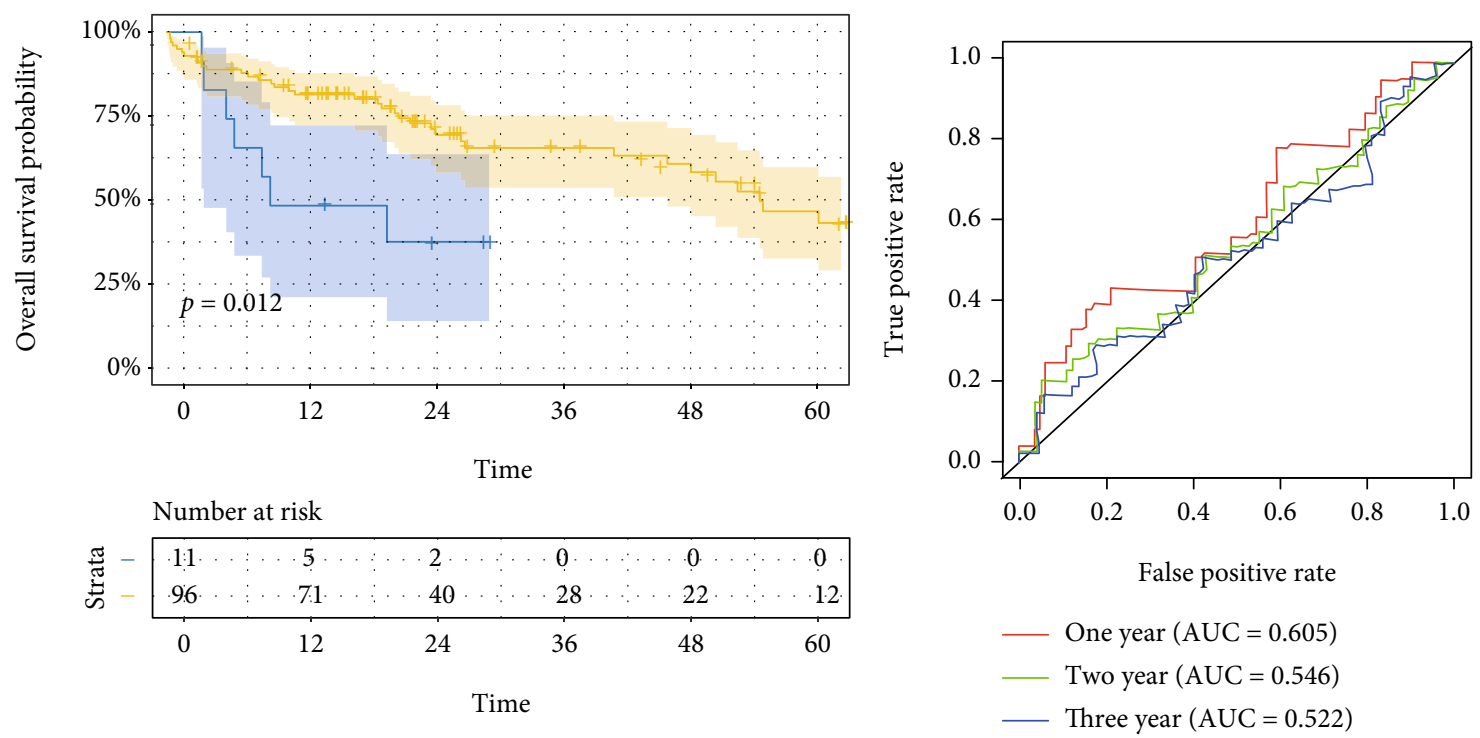

Strata

$$
\begin{aligned}
& + \text { Risk }=\text { high } \\
& + \text { Risk }=\text { low }
\end{aligned}
$$

(c)
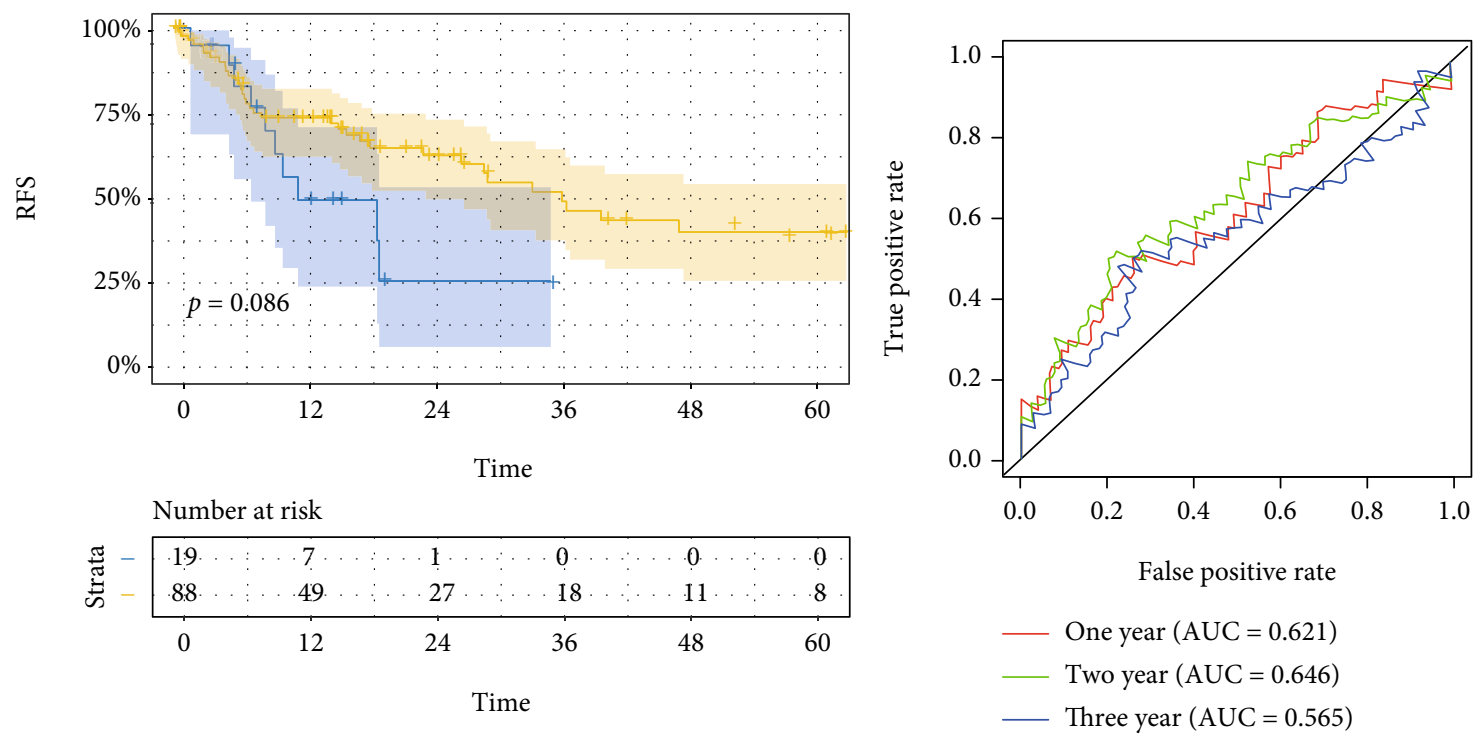

Strata

$$
\begin{aligned}
& + \text { Risk }=\text { high } \\
& + \text { Risk }=\text { low }
\end{aligned}
$$

(d)

FIGURE 2: Validation of two prognostic models. Kaplan-Meier survival curve and ROC curve for OS and RFS in the training set (a, b) and the validation set $(c, d)$.

training set and validation set. In the training set, KaplanMeier curves showed the favorable prognosis of OS and RFS in the low-risk group (Figures 2(a) and 2(b)). Besides, ROC curves were performed for prediction for OS and RFS by using above prognostic model, and the area under the curve (AUC) values for OS at 1,3 , and 5 years were 0.605 , 0.546 , and 0.522 , and AUC values for RFS at 1,3 , and 5 years were $0.621,0.646$, and 0.565 , respectively. The ROC curves and AUC values in both prognostic models indicated the dis- criminative value in both prognostic models. In line with above results, the Kaplan-Meier curves from the validation set also showed the prognosis of OS and RFS was dismal in the high-risk group. The satisfactory AUC values also verified the predictive ability of two signatures (Figures 2(c) and 2(d)).

3.3. Establishment of mRNA-lncRNA Networks. The Pearson correlation analysis was utilized to established the mRNAlncRNA coexpression network in order to explore the 


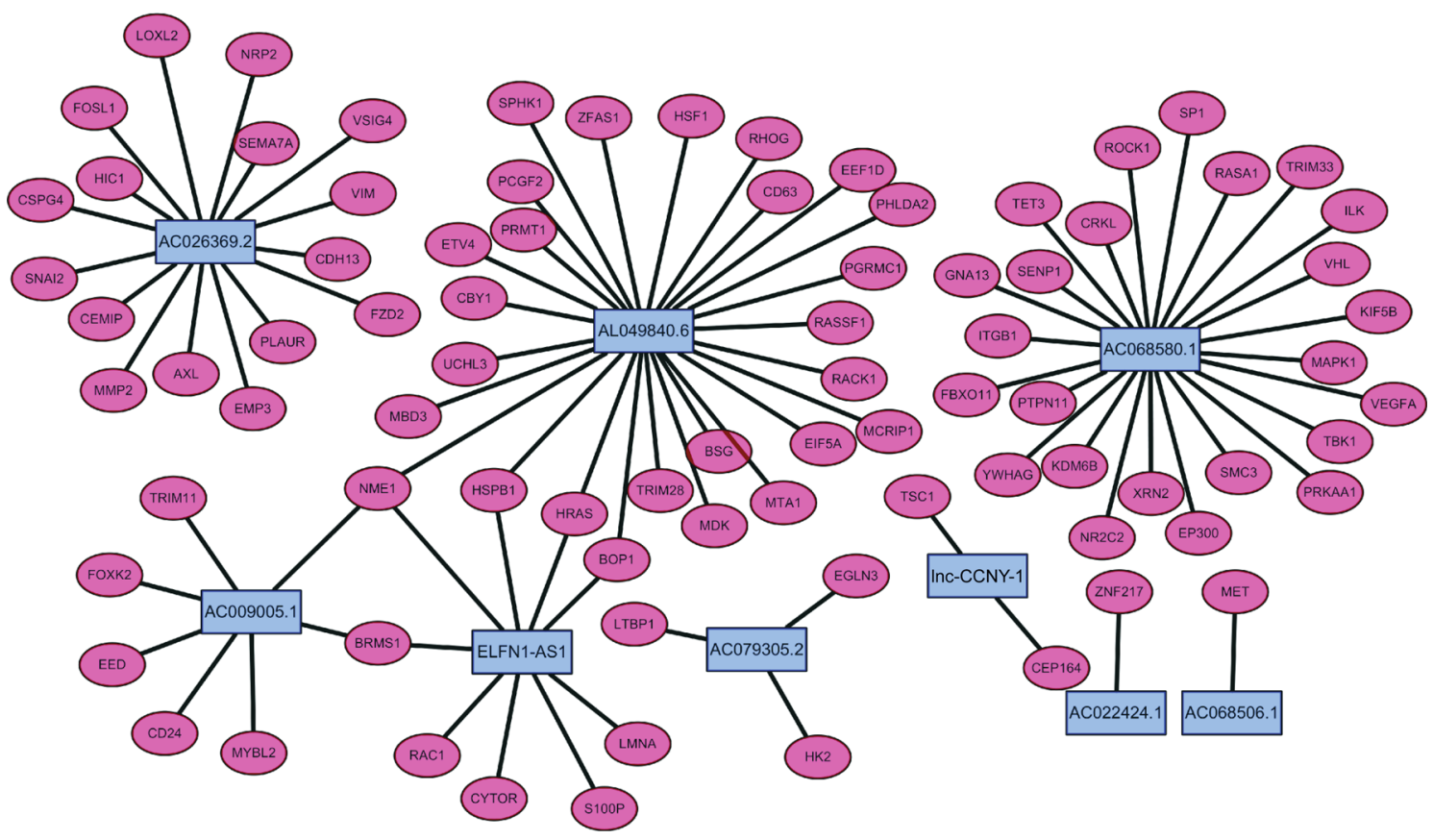

(a)

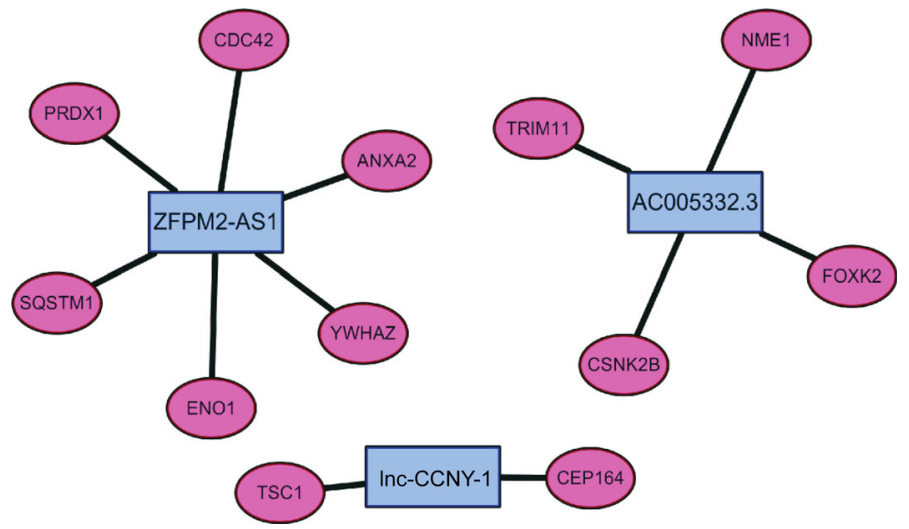

(b)

FIGURE 3: mRNA-lncRNA networks associated with OS signature (a) and RFS signature (b).

regulatory mechanism between IncRNA and mRNA. The Figure 3(a) showed the network between mRNAs and nine EMT-related IncRNAs associated with OS prognosis, and Figure 3(b) displayed the network between mRNAs with three EMT-related IncRNAs related with RFS signature. When we selected the lnc-CCNY-1 which was simultaneously associated with OS and RFS into further analysis, TSC1 and CEP164 were obtained as the target genes. These genes were reserved for future research.

3.4. Characteristics of Gene Set Variation Analysis and Immune Cell Infiltration. Based on the similarity of expression of EMT-related lncRNAs, the $k=2$ was finally selected with optimal clustering stability (Figure 4(a)). A total of 377 patients with HCC were clustered into two subgroups, in cluster $\mathrm{C} 1$ and in cluster $\mathrm{C} 2$. The Kaplan-Meier curves showed that OS (Figure 4(b)) and RFS (Figure 4(c)) of cluster $\mathrm{C} 1$ were longer than those of cluster C2 $(p<0.0001, p$
$=0.00022$, respectively). As shown in Figures 4(d), 4(e), and 4(g), cluster $\mathrm{C} 1$ had higher stromal score, ESTIMATE score, and immune score than cluster C2. The discrepancy of composition of 22 immune cells between two clusters showed that higher $\mathrm{T}$ regulatory cells (Tregs) and lower macrophages M2 were shown in cluster $\mathrm{C} 1$, as presented in Figure 4(h).

3.5. Lnc-CCNY-1 Is the Key EMT-Related IncRNA in HCC. To further investigate the biomarkers for prognosis and tumorigenesis in HCC, we selected the tumorigenesis lncRNA which was simultaneously incorporated into OS prognostic model and RFS prognostic model. The KaplanMeier curves showed that patients with high expression of lnc-CCNY-1 had the unfavorable prognosis in both OS and RFS (Figures 5(a) and 5(b)). GSVA analysis was utilized to further explore the biological function of the key EMTrelated lncRNAs. The further analysis showed that lnc- 

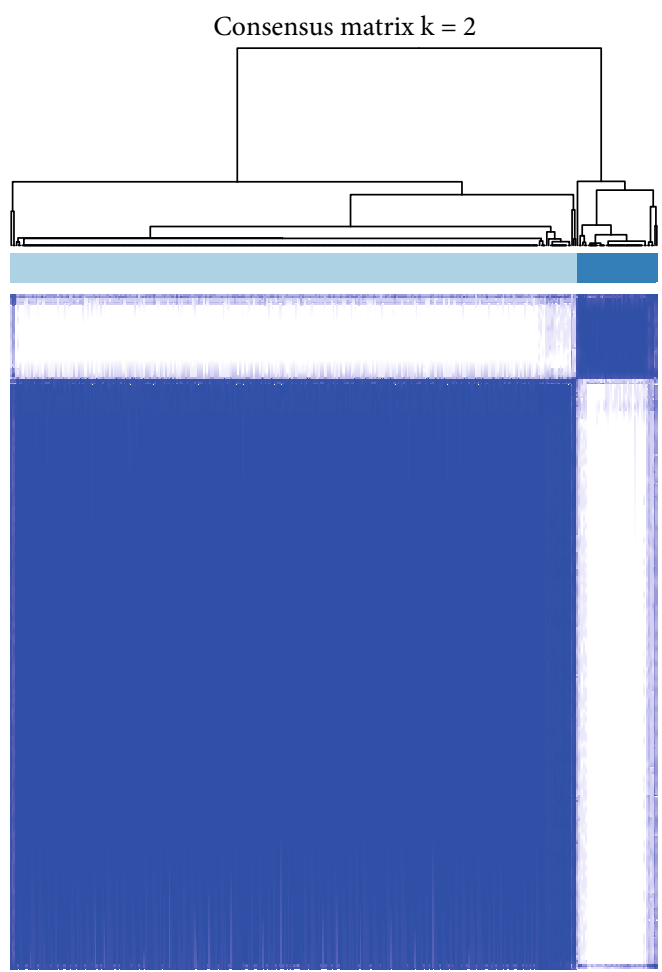

$\square 1$

$\square 2$

(a)

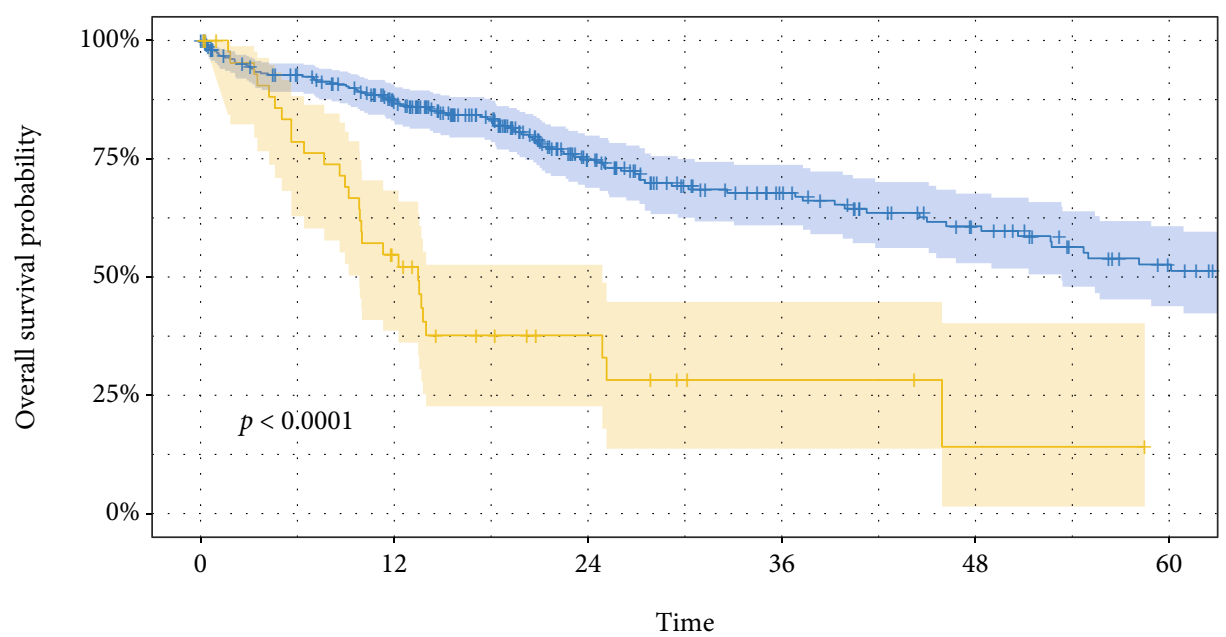

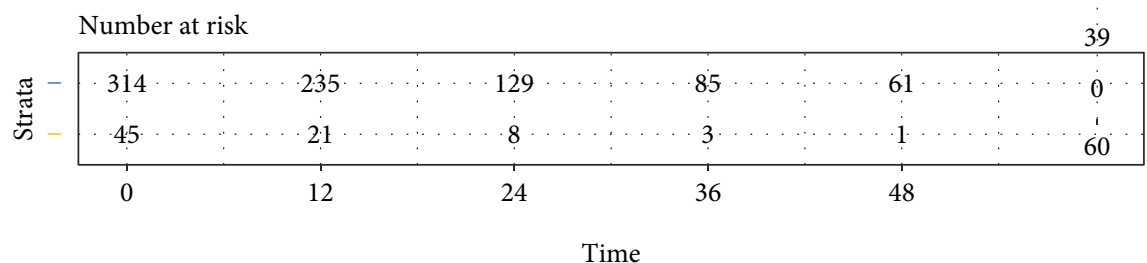

Strata

+ Cluster=Cluster 1

+ Cluster=Cluster2

(b)

Figure 4: Continued. 

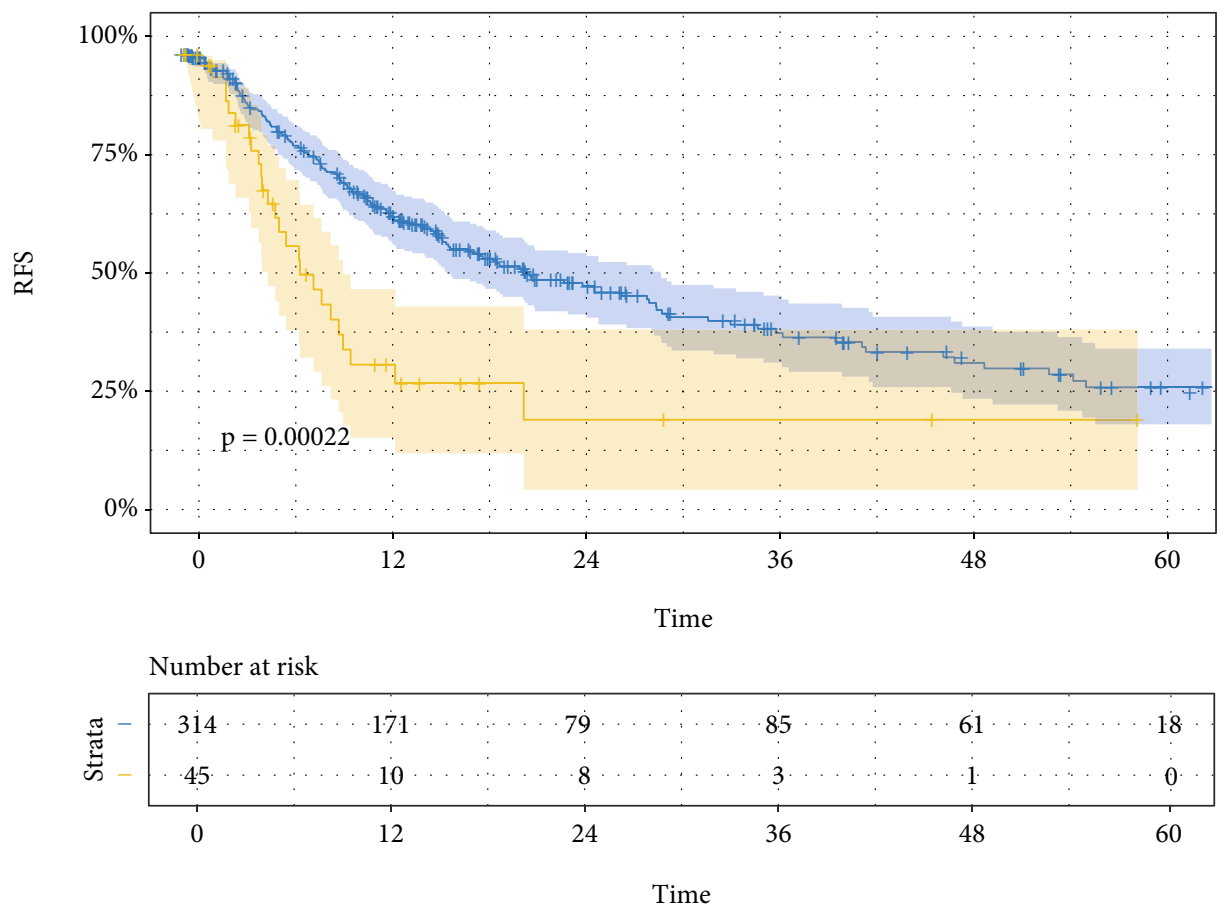

Strata

+ Cluster=Cluster 1

+ Cluster=Cluster2

(c)

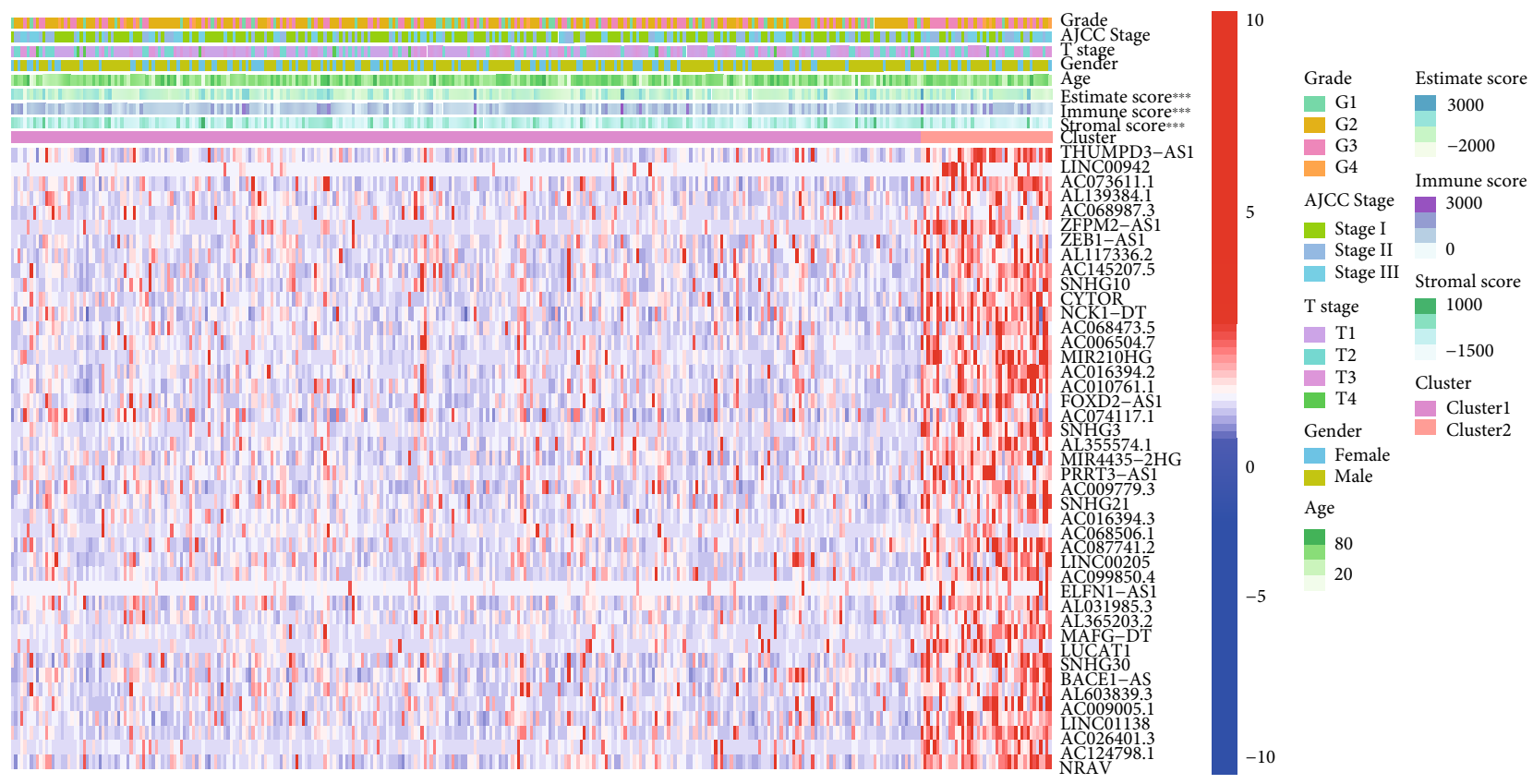

(d)

Figure 4: Continued. 


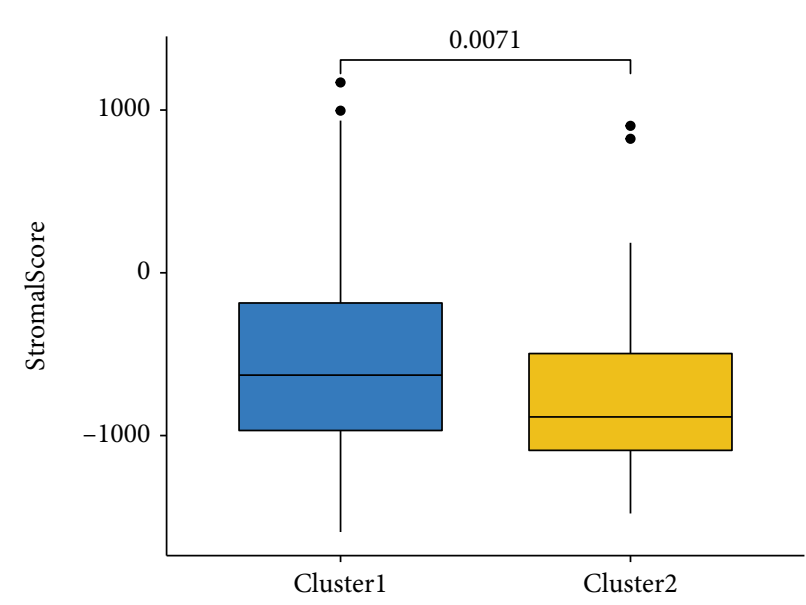

Cluster

Cluster1

투 Cluster2

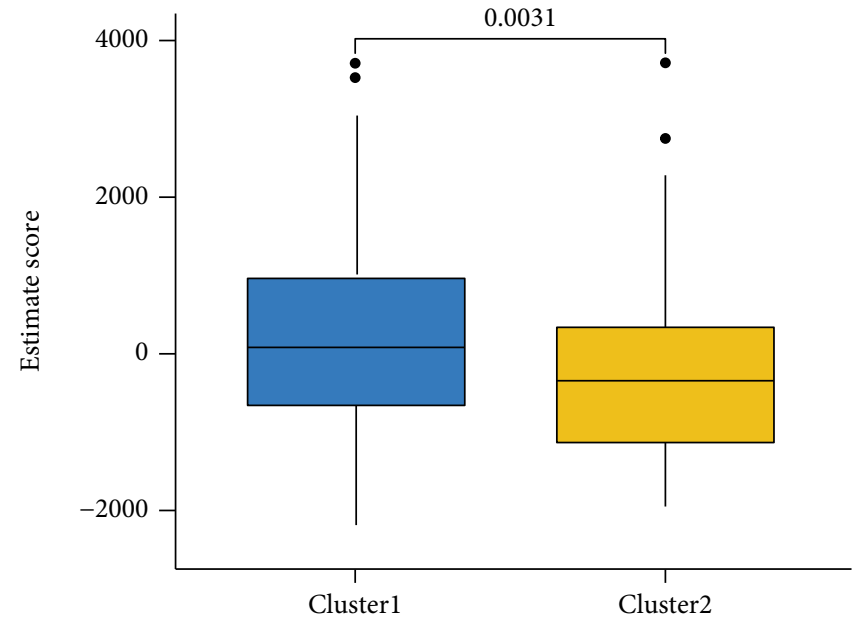

Cluster

官 Cluster1

追 Cluster2

(e)

(f)

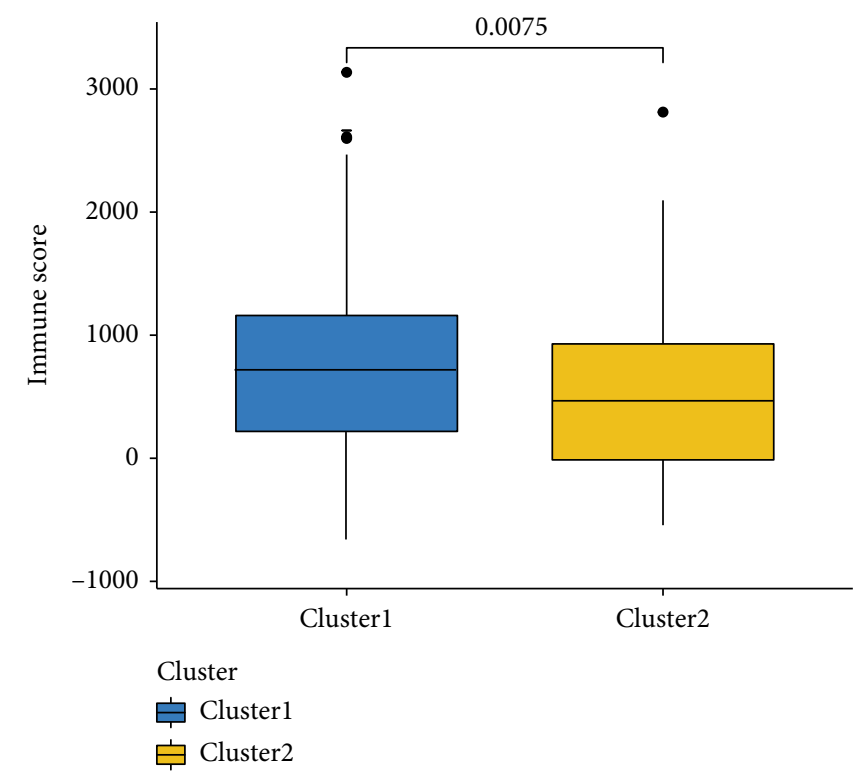

(g)

Figure 4: Continued. 


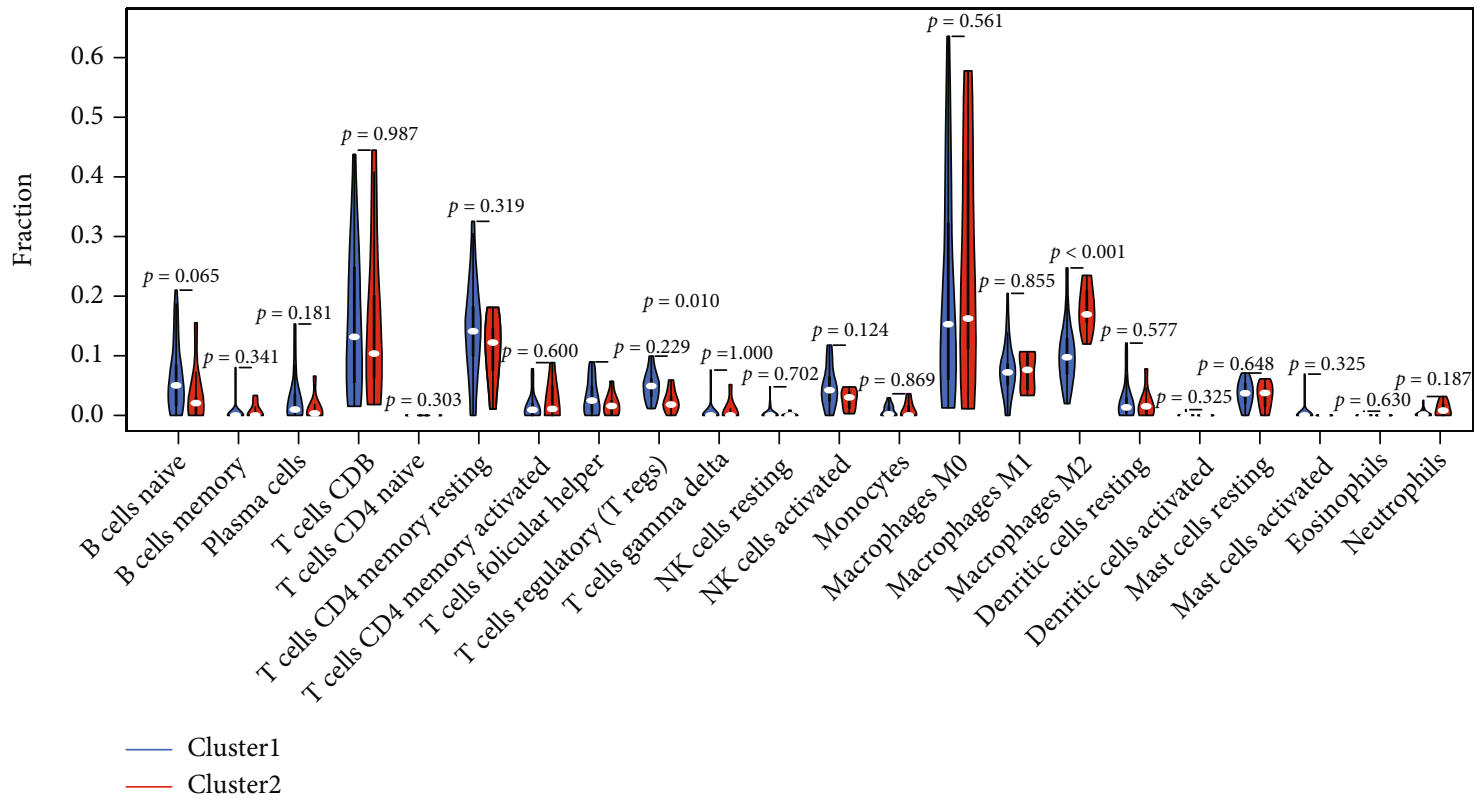

(h)

FIgURE 4: The association of prognosis and immune landscape with EMT-related lncRNAs. Patients with HCC were grouped into two clusters according to the consensus clustering matrix $(k=2)$ (a). Kaplan-Meier survival curve for OS (b) and RFS (c) between two clusters. The heatmap of clinicopathological characters, immune microenvironment, and EMT-related lncRNA expression between two clusters (d). Comparison of stromal score, ESTIMATE score, and immune score between the two clusters (e-g). Difference of infiltrating immune cell between two clusters (h).

CCNY-1 lncRNA was significantly positive associated with cell cycle pathway and negatively associated with peroxisome proliferator-activated receptor (PPAR) signaling pathway, as shown in Figures 6(a) and 6(b).

To further investigate the regulatory mechanism of lncCCNY-1, the radar chart was drawn to show the correlation with immune cells (Figure 7). The expression of lnc-CCNY1 was positively associated with plasma cells, macrophages $\mathrm{M} 0$, and $\mathrm{T}$ cell follicular helper and negatively correlated with CD4 memory-activated macrophages M1.

\section{Discussion}

The pathogenesis of HCC was very complicated because occurrence resulted from several factors, including internal gene factors, external environment, and progress of chronic liver disease [24]. Besides, preliminary investigation also demonstrated that the occurrence and progression of HCC were significantly associated with cell cycle, apoptosis, and energy metabolism abnormalities $[27,28]$. So, it is worth to understand the tumorigenesis and evolution mechanism of HCC due to the dismal prognosis in clinical. The increasing studies showed that EMT and lncRNAs were incorporated into the progression of HCC [29-32]. In our study, two EMT-related lncRNA prognostic models of OS and RFS were constructed. Kaplan-Meier curves showed the favorable prognosis of OS and RFS in the low-risk group. The ROC curves and AUC values in two prognostic models indicated the discriminative value in the training set and validation set.
EMT is a cellular program which played a crucial role for embryogenesis, wound healing, and malignant progression [33]. In this course, the stone epithelial appearance of epithelial cells was lost to adopt a spindle shaped, mesenchymal morphology. During the course of tumor progression, EMT confers on individual carcinoma cell multiple traits in many cancers [34-37]. Previous studies had showed that genes influenced the proliferation, invasion, and metastasis of HCC by regulating EMT procedure [38, 39]. For instance, Meng et al. [6] found that USP5 could accelerate HCC cell proliferation, metastasis, and invasion through coordinate EMT program. Increasing investigations had demonstrated that lncRNAs showed the crucial role in diverse physiological and pathological processes. It was reported [40] that lncRNA MIAT accelerated proliferation and invasion of HCC cells by regulating miR-214. The study performed by Pan et al. demonstrated that lncRNA-PDPK2P promoted HCC progression through the PDK1/AKT/Caspase 3 pathway.

EMT-related lncRNAs were identified by using TCGA database and EMT gene base. Then, we selected the differentially expressed EMT-related lncRNAs by comparing the tumor tissues and adjacent normal tissues. Finally, nine lncRNAs were significantly associated with OS, and three lncRNAs were correlated with RFS. The risk scores of each patient were calculated by the coefficient and expression of aforementioned lncRNAs and further divided the patients with HCC into the high-risk group and low-risk group. In training set, Kaplan-Meier curves showed the favorable prognosis of OS and RFS in the low-risk group. The ROC curves and AUC values in both prognostic models indicated the discriminative value in both prognostic models. In line 


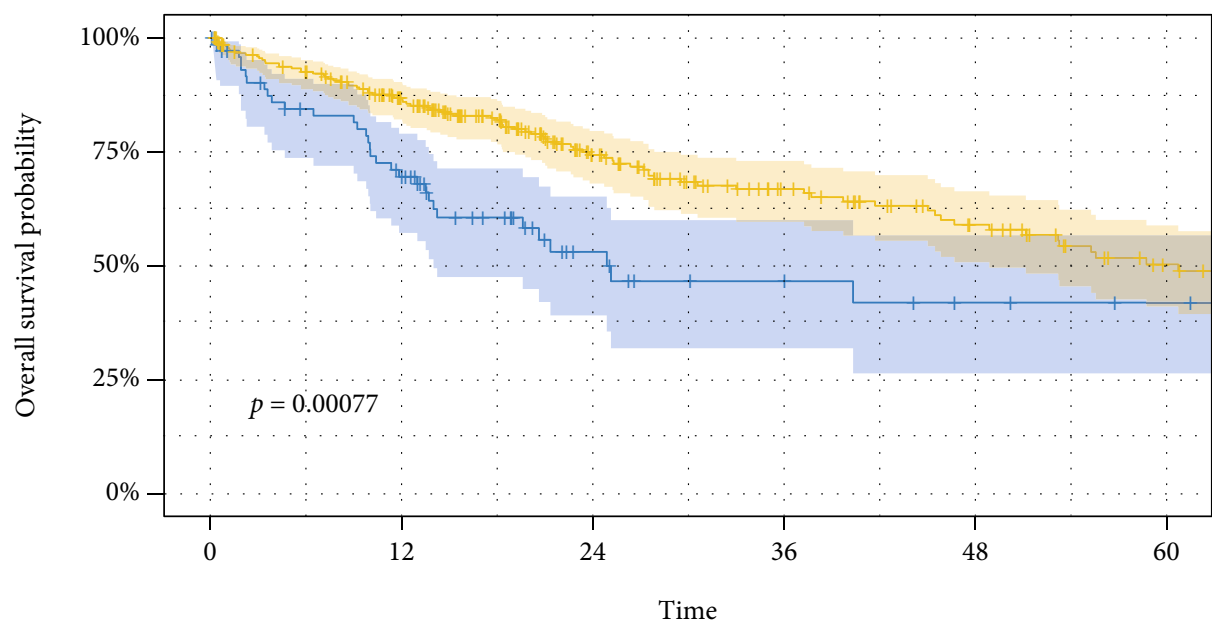

Number at risk

\begin{tabular}{|c|c|c|c|c|c|}
\hline 777 & 46 & 17 & 11 & 7 & 5 \\
\hline 282 & 210 & 120 & 77 & 55 & 34 \\
\hline T & $T$ & T & $T$ & $T$ & 1 \\
\hline 0 & 12 & 24 & 36 & 48 & 60 \\
\hline
\end{tabular}

(a)

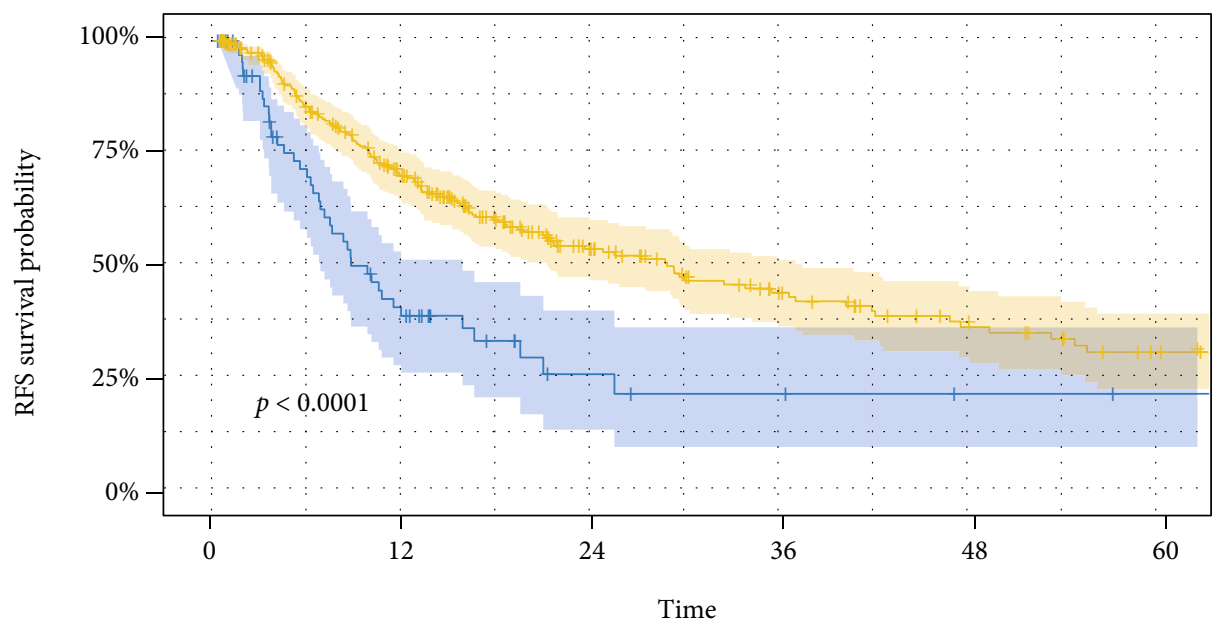

Number at risk

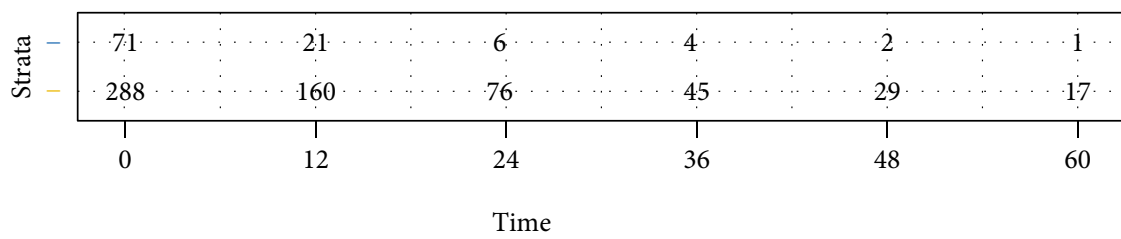

Strata
+ Group=high
+ Group=low

(b)

FIgURE 5: Kaplan-Meier survival curve for OS and RFS, according to the expression of Lnc-CCNY-1.

with the conclusion in the training set, the same results of Kaplan-Meier curves and high accuracy of prognostic models were shown in the validation set.

In recent years, TME had received more attention due the immunotherapy had showed curative effect in cancer management. TME acted a critical role in tumor progression and affected the effect of immunotherapy. To investigate the association between EMT-related lncRNAs with TME, "Consensus Cluster Plus" package was used to generate two clusters of HCC cohort. The Kaplan-Meier curves in 


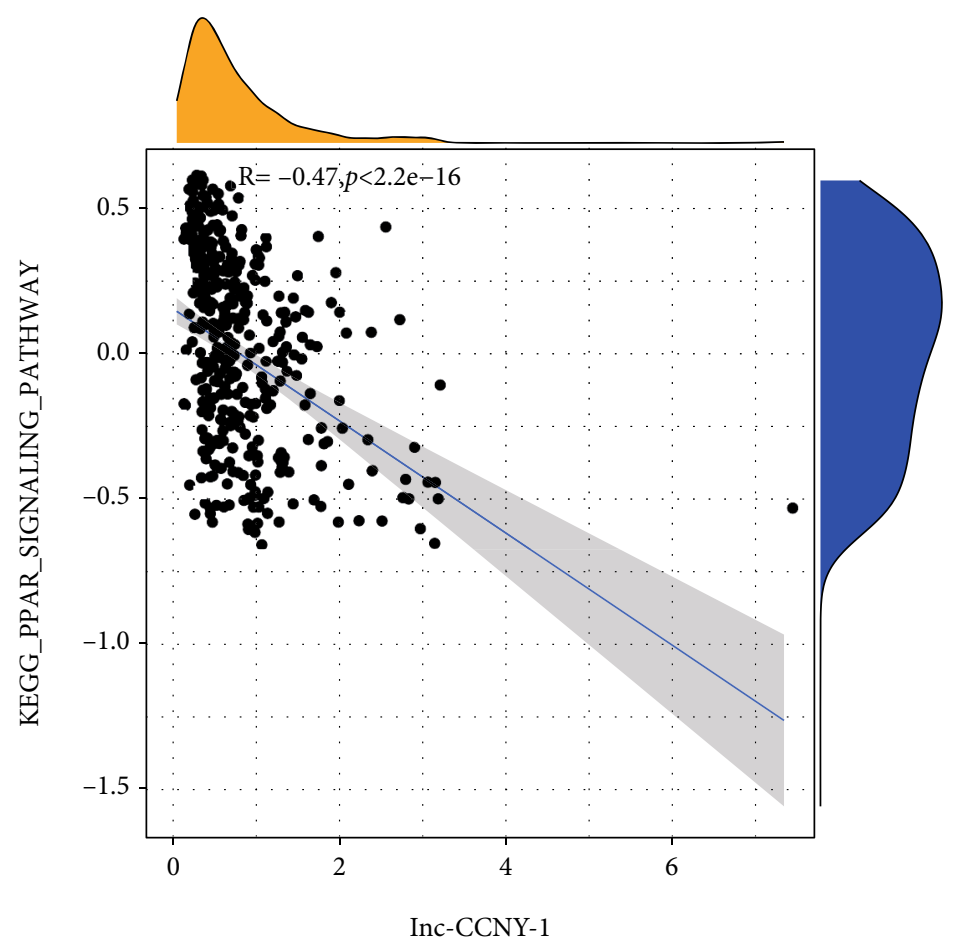

(a)

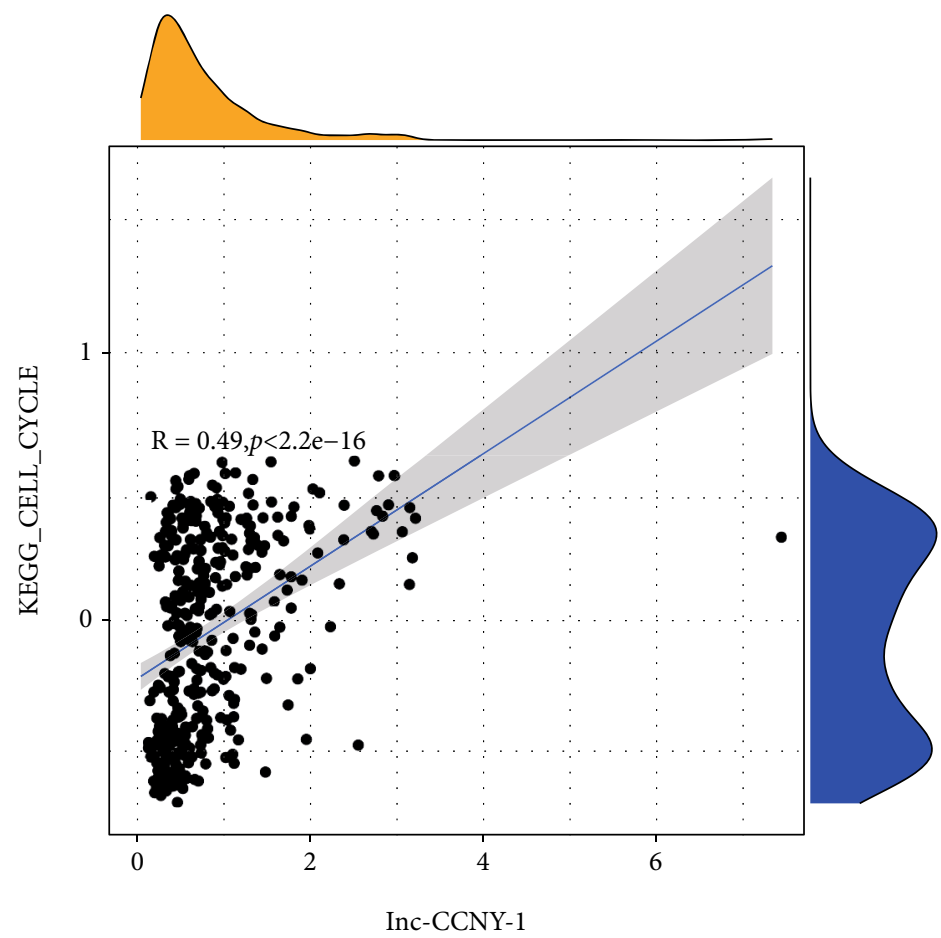

(b)

Figure 6: The relationship of Lnc-CCNY-1 and pathways.

our study showed that OS and RFS of cluster C1 were longer than those of cluster C2. The differences between two clusters in terms of stromal score, ESTIMATE score and immune score, indicate that EMT-related lncRNAs may affect tumor progression by regulating TME. A previous study performed by Peng et al. [41] demonstrated that
LncRNA MIAT was significantly associated with immune escape of HCC and altered the immune cell infiltration. Ji et al. [42] reported that Lnc-Tim3 was significant with $\mathrm{CD} 8+\mathrm{T}$ exhaustion and the survival of the exhausted CD8 $+\mathrm{T}$ cells. Our results showed that the higher expression of Tregs and lower expression of macrophages M2 were shown 


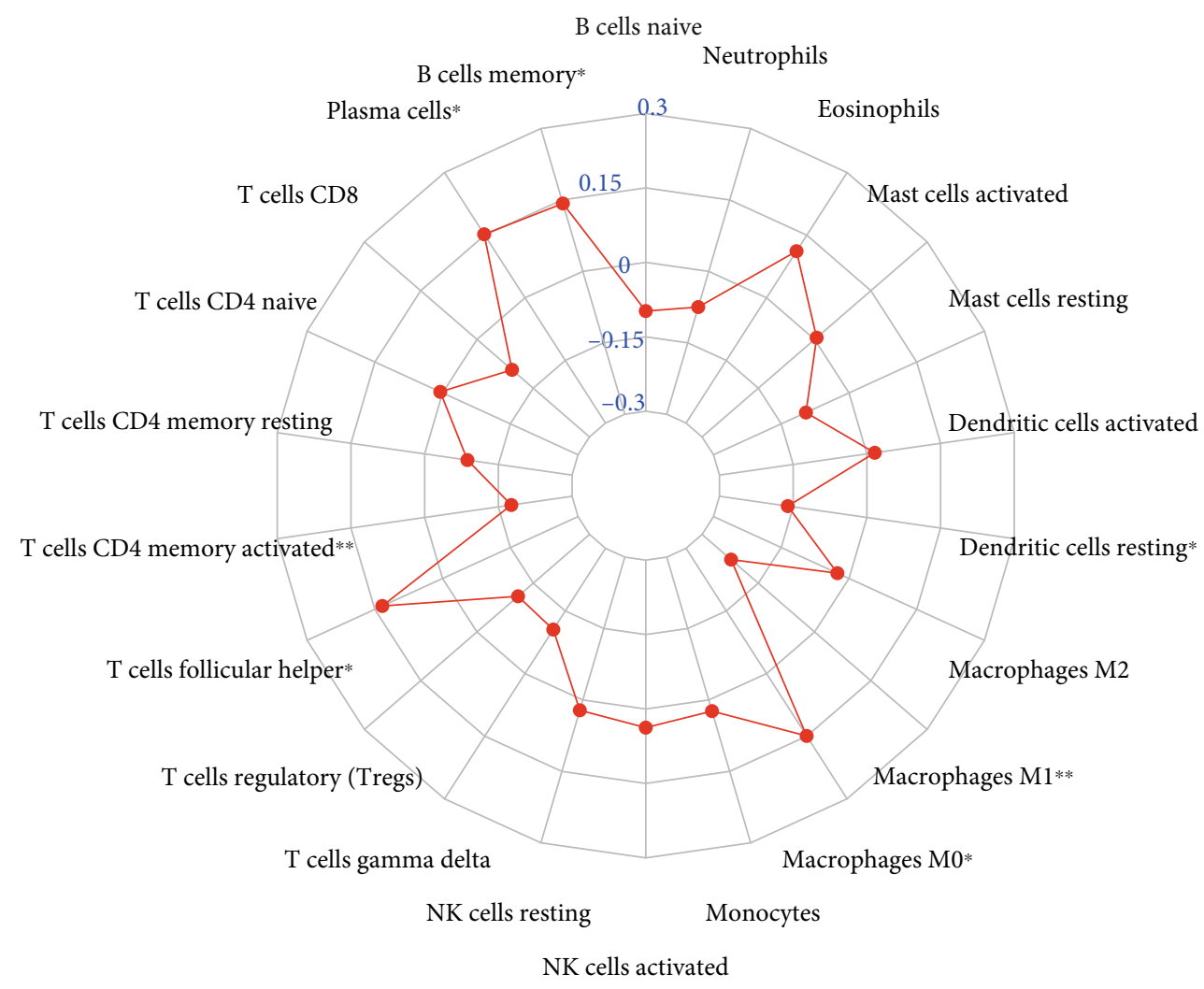

Figure 7: Correlation between immune cells and the expression of Lnc-CCNY-1 ( ${ }^{*}$ indicated $p<0.05,{ }^{* *}$ indicated $\left.p<0.01\right)$.

in cluster $\mathrm{C} 1$ which had favorable prognosis, with the comparison with cluster 2 . Tregs which mainly derived from peripheral blood $\mathrm{T}$ lymphocytes were significantly correlated with HCC invasiveness and played important roles in hampering effect of antitumor responses in HCC [43-45]. It had been reported that activated Tregs prohibit different types of immune cells by contact-dependent interactions and further contribute to immune dysfunction in HCC [46]. M2polarized macrophages characterized as tumor-associated macrophages (TAMs) were the important composition of tumor-infiltrating immune cells. The study by Yao et al. reported that M2-polarized macrophages accelerated the migration and invasion of HCC cells via EMT process [47]. The regulatory mechanism of TME had not been elucidated and is worthy to investigate in future research.

To further investigate the biomarkers for prognosis and tumorigenesis in HCC, lnc-CCNY-1 which was simultaneously incorporated into OS prognostic model and RFS prognostic model was identified in our research. The expression of lnc-CCNY-1 was negatively with progression in terms for OS and RFS, indicating lnc-CCNY-1 may promote tumor progression of HCC. GSVA analysis showed that lncCCNY-1 was negatively associated with PPAR signaling pathway and positively correlated with CELL cycle pathway. Previous studies had reported that PPAR signaling pathway was downregulated in colorectal cancer and non-small-cell lung cancer [48, 49]. However, there was no investigation about PPAR signaling pathway in HCC, which is reserved for further research. Cell cycle pathway was a critical mechanism, by which many genes could promote HCC cell proliferation [50-52]. The reasonable explanation was that lnc-CCNY-1 promote tumor progression of HCC by downregulating PPAR signaling pathway and upregulating cell cycle.

It was noted that our study also included several limitations. Firstly, the EMT-related lncRNA prognostic model was generated by bioinformatic approaches, which is not validated in our registers. Besides, lnc-CCNY-1 was identified as the key EMT-related lncRNA, which was not verified in in vivo or further in vitro experiment in this study. We plan to further investigate the mechanism of lnc-CCNY-1 for HCC in next research.

\section{Conclusion}

Two EMT-related lncRNA prognostic models of OS and RFS were constructed to discriminate patients and predict prognosis of HCC. EMT-related lncRNAs may play a role on prognosis of HCC by influencing the immune microenvironment. Lnc-CCNY-1 was selected as the key EMT-related lncRNA for further research.

\section{Data Availability}

The data used to support the findings of this study are included within the article and the supplementary information files. 


\section{Conflicts of Interest}

The authors have no conflicts of interest to declare.

\section{Authors' Contributions}

Z.Y and J. L contributed to study concepts and design. Y. Z and $\mathrm{L}$. W contributed to data acquisition, analysis, and interpretation. W. Z, J. M, and Z. Z contributed to data analysis and statistical analysis. M. Y and J. Y contributed to quality control of data and algorithms. Y. Z contributed to manuscript editing. Z. Y and J. L contributed to manuscript review. Yongjie Zhou and Liangwen Wang contributed equally to this work.

\section{Acknowledgments}

This study was supported by the National Natural Science Foundation of China (81771948).

\section{Supplementary Materials}

Supplementary 1. Supplementary Table 1: the differentially expressed EMT-related lncRNAs between tumor tissues with adjacent normal tissues.

Supplementary 2. Supplementary Table 2: 492 EMT-related IncRNAs associated with OS signature screened by the univariate Cox regression analysis.

Supplementary 3. Supplementary Table 3: 480 EMT-related IncRNAs associated with RFS signature screened by the univariate Cox regression analysis.

\section{References}

[1] J. J. Harding, G. Abu-Zeinah, J. F. Chou et al., "Frequency, morbidity, and mortality of bone metastases in advanced hepatocellular carcinoma," Journal of the National Comprehensive Cancer Network: JNCCN, vol. 16, no. 1, pp. 50-58, 2018.

[2] J. J. Shaw and S. A. Shah, "Rising incidence and demographics of hepatocellular carcinoma in the USA: what does it mean?," Expert Review of Gastroenterology \& Hepatology, vol. 5, no. 3, pp. 365-370, 2011.

[3] European Association For The Study Of The Liver, "EASL clinical practice guidelines: management of hepatocellular carcinoma," Journal of Hepatology, vol. 69, no. 1, pp. 182-236, 2018.

[4] G. Giannelli, P. Koudelkova, F. Dituri, and W. Mikulits, "Role of epithelial to mesenchymal transition in hepatocellular carcinoma," Journal of Hepatology, vol. 65, no. 4, pp. 798-808, 2016.

[5] Y. Dong, Q. Zheng, Z. Wang et al., "Higher matrix stiffness as an independent initiator triggers epithelial-mesenchymal transition and facilitates HCC metastasis," Journal of Hematology \& Oncology, vol. 12, no. 1, p. 112, 2019.

[6] J. Meng, X. Ai, Y. Lei et al., "USP5 promotes epithelialmesenchymal transition by stabilizing SLUG in hepatocellular carcinoma," Theranostics, vol. 9, no. 2, pp. 573-587, 2019.

[7] J. Zhang, Q. Zhang, Y. Lou et al., "Hypoxia-inducible factor$1 \alpha /$ interleukin- $1 \beta$ signaling enhances hepatoma epithelial- mesenchymal transition through macrophages in a hypoxicinflammatory microenvironment," Hepatology (Baltimore, $M d$ ), vol. 67, no. 5, pp. 1872-1889, 2018.

[8] B. Wang, T. Liu, J. C. Wu et al., "STAT3 aggravates TGF- $\beta 1$ induced hepatic epithelial-to-mesenchymal transition and migration," Biomedicine \& Pharmacotherapy = Biomedecine \& pharmacotherapie, vol. 98, pp. 214-221, 2018.

[9] A. M. Schmitt and H. Y. Chang, "Long noncoding RNAs in cancer pathways," Cancer Cell, vol. 29, no. 4, pp. 452-463, 2016.

[10] E. Leucci, R. Vendramin, M. Spinazzi et al., "Melanoma addiction to the long non-coding RNA SAMMSON," Nature, vol. 531, no. 7595, pp. 518-522, 2016.

[11] J. H. Yuan, X. N. Liu, T. T. Wang et al., "The MBNL3 splicing factor promotes hepatocellular carcinoma by increasing PXN expression through the alternative splicing of lncRNA-PXN-AS1," Nature Cell Biology, vol. 19, no. 7, pp. 820-832, 2017.

[12] D. Xu, F. Yang, J. H. Yuan et al., "Long noncoding RNAs associated with liver regeneration 1 accelerates hepatocyte proliferation during liver regeneration by activating $\mathrm{Wnt} / \beta$-catenin signaling," Hepatology (Baltimore, $M d$ ), vol. 58, no. 2, pp. 739-751, 2013.

[13] C. Zhang, J. Yuan, H. Hu et al., "Long non-coding RNA CHCHD4P4 promotes epithelial-mesenchymal transition and inhibits cell proliferation in calcium oxalate-induced kidney damage," Brazilian Journal of Medical and Biological Research, vol. 51, no. 1, p. e6536, 2018.

[14] X. Fu, X. Zhu, F. Qin et al., "Linc00210 drives Wnt/ $\beta$-catenin signaling activation and liver tumor progression through CTNNBIP1-dependent manner," Molecular cancer., vol. 17, no. 1, p. 73, 2018.

[15] W. C. Liang, J. L. Ren, C. W. Wong et al., "LncRNA-NEF antagonized epithelial to mesenchymal transition and cancer metastasis via cis-regulating FOXA2 and inactivating $\mathrm{Wnt} / \beta$ catenin signaling," Oncogene, vol. 37 , no. 11, pp. 1445-1456, 2018.

[16] X. Yan, D. Zhang, W. Wu et al., "Mesenchymal stem cells promote hepatocarcinogenesis via lncRNA-MUF interaction with ANXA2 and miR-34a," Cancer Research, vol. 77, no. 23, pp. 6704-6716, 2017.

[17] T. Y. Seiwert, B. Burtness, R. Mehra et al., "Safety and clinical activity of pembrolizumab for treatment of recurrent or metastatic squamous cell carcinoma of the head and neck (KEYNOTE-012): an open-label, multicentre, phase $1 \mathrm{~b}$ trial," The Lancet Oncology, vol. 17, no. 7, pp. 956-965, 2016.

[18] M. A. Curran, W. Montalvo, H. Yagita, and J. P. Allison, "PD-1 and CTLA-4 combination blockade expands infiltrating T cells and reduces regulatory $\mathrm{T}$ and myeloid cells within B16 melanoma tumors," Proceedings of the National Academy of Sciences of the United States of America, vol. 107, no. 9, pp. 4275-4280, 2010.

[19] C. Robert, A. Ribas, J. D. Wolchok et al., “Anti-programmeddeath-receptor-1 treatment with pembrolizumab in ipilimumab-refractory advanced melanoma: a randomised dose-comparison cohort of a phase 1 trial," Lancet (London, England), vol. 384, no. 9948, pp. 1109-1117, 2014.

[20] Y. Yuan, "Spatial heterogeneity in the tumor microenvironment," Cold Spring Harbor Perspectives in Medicine, vol. 6, no. $8,2016$. 
[21] A. E. Denton, E. W. Roberts, and D. T. Fearon, "Stromal cells in the tumor microenvironment," Adv. Exp. Med. Biol., vol. 1060, pp. 99-114, 2018.

[22] K. C. Barry, J. Hsu, M. L. Broz et al., “A natural killer-dendritic cell axis defines checkpoint therapy-responsive tumor microenvironments," Nature medicine., vol. 24, no. 8, pp. 11781191, 2018.

[23] D. Lambrechts, E. Wauters, B. Boeckx et al., "Phenotype molding of stromal cells in the lung tumor microenvironment," Nature medicine., vol. 24, no. 8, pp. 1277-1289, 2018.

[24] J. Zucman-Rossi, A. Villanueva, J. C. Nault, and J. M. Llovet, "Genetic landscape and biomarkers of hepatocellular carcinoma," Gastroenterology, vol. 149, no. 5, pp. 1226-1239.e4, 2015.

[25] K. Yoshihara, M. Shahmoradgoli, E. Martínez et al., "Inferring tumour purity and stromal and immune cell admixture from expression data," Nature Communications, vol. 4, no. 1, p. 2612, 2013.

[26] A. M. Newman, C. B. Steen, C. L. Liu et al., "Determining cell type abundance and expression from bulk tissues with digital cytometry," Nature Biotechnology, vol. 37, no. 7, pp. 773$782,2019$.

[27] L. Lin, Z. Yao, K. Bhuvaneshwar et al., "Transcriptional regulation of STAT3 by SPTBN1 and SMAD3 in HCC through cAMP-response element-binding proteins ATF3 and CREB2," Carcinogenesis, vol. 35, no. 11, pp. 2393-2403, 2014.

[28] C. C. Hsu, L. C. Wu, C. Y. Hsia et al., "Energy metabolism determines the sensitivity of human hepatocellular carcinoma cells to mitochondrial inhibitors and biguanide drugs," Oncology Reports, vol. 34, no. 3, pp. 1620-1628, 2015.

[29] C. Xiao, X. Wan, H. Yu et al., "LncRNA-AB209371 promotes the epithelial-mesenchymal transition of hepatocellular carcinoma cells," Oncology Reports, vol. 41, no. 5, pp. 2957-2966, 2019.

[30] B. W. Chen, Y. Zhou, T. Wei et al., "IncRNA-POIR promotes epithelial-mesenchymal transition and suppresses sorafenib sensitivity simultaneously in hepatocellular carcinoma by sponging miR-182-5p," Journal of Cellular Biochemistry, vol. 122, no. 1, pp. 130-142, 2021.

[31] Y. Wang, Z. Liu, B. Yao et al., "Long non-coding RNA CASC2 suppresses epithelial-mesenchymal transition of hepatocellular carcinoma cells through CASC2/miR-367/FBXW7 axis," Molecular Cancer, vol. 16, no. 1, p. 123, 2017.

[32] E. Changyong, J. Yang, H. Li, and C. Li, "LncRNA LOC105372579 promotes proliferation and epithelialmesenchymal transition in hepatocellular carcinoma via activating miR-4316/FOXP4 signaling," Cancer Management and Research, vol. 11, pp. 2871-2879, 2019.

[33] A. Dongre and R. A. Weinberg, "New insights into the mechanisms of epithelial-mesenchymal transition and implications for cancer," Nature Reviews Molecular Cell Biology, vol. 20, no. 2, pp. 69-84, 2019.

[34] A. D. Rhim, E. T. Mirek, N. M. Aiello et al., "EMT and dissemination precede pancreatic tumor formation," Cell, vol. 148, no. 1-2, pp. 349-361, 2012.

[35] A. M. Krebs, J. Mitschke, M. Lasierra Losada et al., "The EMTactivator Zeb1 is a key factor for cell plasticity and promotes metastasis in pancreatic cancer," Nature Cell Biology, vol. 19, no. 5, pp. 518-529, 2017.

[36] S. A. Mani, W. Guo, M. J. Liao et al., "The epithelialmesenchymal transition generates cells with properties of stem cells," Cell, vol. 133, no. 4, pp. 704-715, 2008.
[37] A. Singh and J. Settleman, "EMT, cancer stem cells and drug resistance: an emerging axis of evil in the war on cancer," Oncogene, vol. 29, no. 34, pp. 4741-4751, 2010.

[38] H. Liu, Y. Ma, H. W. He, W. L. Zhao, and R. G. Shao, "SPHK1 (sphingosine kinase 1) induces epithelial-mesenchymal transition by promoting the autophagy-linked lysosomal degradation of CDH1/E-cadherin in hepatoma cells," Autophagy, vol. 13, no. 5, pp. 900-913, 2017.

[39] Q. Zhai, J. Qin, X. Jin et al., "PADI4 modulates the invasion and migration of osteosarcoma cells by down-regulation of epithelial-mesenchymal transition," Life Sciences, vol. 256, article 117968,2020

[40] X. Huang, Y. Gao, J. Qin, and S. Lu, "IncRNA MIAT promotes proliferation and invasion of HCC cells via sponging miR-214," American Journal of Physiology Gastrointestinal and Liver Physiology, vol. 314, no. 5, pp. G559-G565, 2018.

[41] L. Peng, Y. Chen, Q. Ou, X. Wang, and N. Tang, "LncRNA MIAT correlates with immune infiltrates and drug reactions in hepatocellular carcinoma," International Immunopharmacology, vol. 89, article 107071, Part A, 2020.

[42] J. Ji, Y. Yin, H. Ju et al., "Long non-coding RNA Lnc-Tim3 exacerbates CD8 $\mathrm{T}$ cell exhaustion via binding to Tim-3 and inducing nuclear translocation of Bat3 in HCC," Cell Death \& Disease, vol. 9, no. 5, p. 478, 2018.

[43] Y. Fu, S. Liu, S. Zeng, and H. Shen, "From bench to bed: the tumor immune microenvironment and current immunotherapeutic strategies for hepatocellular carcinoma," Journal of Experimental \& Clinical Cancer Research: CR, vol. 38, no. 1, p. 396, 2019.

[44] S. Kalathil, A. A. Lugade, A. Miller, R. Iyer, and Y. Thanavala, "Higher frequencies of GARP(+)CTLA-4(+)Foxp3(+) T regulatory cells and myeloid-derived suppressor cells in hepatocellular carcinoma patients are associated with impaired T-cell functionality," Cancer Research, vol. 73, no. 8, pp. 24352444, 2013.

[45] Q. Gao, S. J. Qiu, J. Fan et al., "Intratumoral balance of regulatory and cytotoxic T cells is associated with prognosis of hepatocellular carcinoma after resection," Journal of Clinical Oncology: official journal of the American Society of Clinical Oncology, vol. 25, no. 18, pp. 2586-2593, 2007.

[46] B. Langhans, H. D. Nischalke, B. Krämer et al., "Role of regulatory $\mathrm{T}$ cells and checkpoint inhibition in hepatocellular carcinoma," Cancer Immunology, Immunotherapy: CII, vol. 68, no. 12, pp. 2055-2066, 2019.

[47] R. R. Yao, J. H. Li, R. Zhang, R. X. Chen, and Y. H. Wang, "M2polarized tumor-associated macrophages facilitated migration and epithelial-mesenchymal transition of HCC cells via the TLR4/STAT3 signaling pathway," World Journal of Surgical Oncology, vol. 16, no. 1, p. 9, 2018.

[48] J. Chen, A. Liu, Z. Lin et al., "Downregulation of the circadian rhythm regulator HLF promotes multiple-organ distant metastases in non-small cell lung cancer through PPAR/NF- $\kappa \mathrm{b}$ signaling," Cancer Letters, vol. 482, pp. 56$71,2020$.

[49] X. Zhang, J. Yao, H. Shi, B. Gao, and L. Zhang, "LncRNATINCR/microRNA-107/CD36 regulates cell proliferation and apoptosis in colorectal cancer via PPAR signaling pathway based on bioinformatics analysis," Biological Chemistry, vol. 400, no. 5, pp. 663-675, 2019.

[50] L. Tuo, J. Xiang, X. Pan et al., "PCK1 negatively regulates cell cycle progression and hepatoma cell proliferation via the 
AMPK/p27Kip1 axis," Journal of Experimental \& Clinical Cancer Research: CR, vol. 38, no. 1, p. 50, 2019.

[51] H. A. Lee, K. B. Chu, E. K. Moon, S. S. Kim, and F. S. Quan, "Sensitization to oxidative stress and G2/M cell cycle arrest by histone deacetylase inhibition in hepatocellular carcinoma cells," Free Radical Biology \& Medicine, vol. 147, pp. 129$138,2020$.

[52] X. H. Liang, Z. P. Feng, F. Q. Liu et al., "MAPRE1 promotes cell cycle progression of hepatocellular carcinoma cells by interacting with CDK2," Cell Biology International, vol. 44, no. 11, pp. 2326-2333, 2020. 